Ziping Wang*, Yangchun Ye, Zhujie Bao and Hao Ge

\title{
Optimization and simulation analysis of structure parameters of OPCM ultrasonic longitudinal wave actuating element
}

https://doi.org/10.1515/secm-2017-0394

Received December 2, 2017; accepted June 13, 2018

\begin{abstract}
The force-electric coupling relationship of the mechanical and electrical properties of piezoelectric composites has been the main factor in the research and development of piezoelectric composites in practical application. A novel orthotropic piezoelectric composite material (OPCM) element is studied in this paper. The properties of the piezoelectric phase and the polymer phase and the influence of the geometrical dimensions of the OPCM on the longitudinal wave drive element are analyzed from the perspective of mechanics and electric power, respectively, and the structural design is optimized. This provides a theoretical basis for the development of OPCM and of new longitudinal ultrasonic phased array actuators.
\end{abstract}

Keywords: actuator; parameter analysis; piezoelectric composite material; structure optimization.

\section{Introduction}

At present, the key material for the conventional phased array transmitting and receiving devices is an ordinary piezoelectric ceramic material. There are many deficiencies in piezoelectric ceramics. The design performance of driving index is poor. Thickness direction and radial electromechanical coupling coefficient have little differences and easily lead to reduced efficiency of the transducer; the piezoelectric ceramic, because of brittleness, is so easily damaged during use, cutting, machining, and assembly

\footnotetext{
*Corresponding author: Ziping Wang, Faculty of Civil Engineering and Mechanics, Jiangsu University, National Center for International Research on Structural Health Management of Critical Components, Zhenjiang, 212013, China, Tel.: +13914559500,

e-mail: wzpxx2004@126.com

Yangchun Ye, Zhujie Bao and Hao Ge: Faculty of Civil Engineering and Mechanics, Jiangsu University, National Center for International Research on Structural Health Management of Critical Components, Zhenjiang, 212013, China
}

and also easily broken under stress. The force and electrical characteristics of a single piezoelectric material are susceptible to temperature, magnetic field, and electric field, which make piezoelectric materials have limited transducer sensitivity and imaging accuracy in nondestructive testing applications of engineering structures $[1,2]$. Therefore, how to research and analyze a novel piezoelectric composite transducer with directional transmitting and receiving functions is of great significance for improving detection sensitivity and signal-to-noise ratio in structural damage detection.

Piezoelectric composite materials have been developed for more than 30 years. In 1978, Newnham, who worked at the United States Pennsylvania State University Materials Laboratory [3], first proposed the concept of piezoelectric composite materials and began to study the application of piezoelectric composite materials in water, then successfully developed the 1-3-type piezoelectric composite in 1980 [4]. Based on piezoelectric sensors, the conclusion that piezoelectric composites outperform monolithic piezoelectric ceramics is demonstrated. In 1989, Chan and others studied the theoretical model of 1-3 piezoelectric composites and the relationship between the effective characteristic parameters and the volume ratio of piezoelectric phases [5]. In 1996, Safai et al. also studied 1-3 piezoelectric composite materials and confirmed that the main characteristic parameters of piezoelectric composites (such as flexibility, coupling with the host, etc.) were higher than those of monolithic piezoelectric ceramics [6]. From the 1990s to the early 21st century, T. Hauke of Germany, Hiroshi Takeuchi of Japan, and G. Hayward [7] and R. Hamilton [8] of the United Kingdom made corresponding transducers with piezoelectric composites. At present, piezoelectric composite materials have been widely used in engineering the structure of health-monitoring technology, which is one of the most widely used fields of intelligent composites. American scientist Bent proposed the concept of piezoelectric fiber composite materials and gradually extended to the field of composite materials in the drive and sensing performance test [9-12]. However, when the piezoelectric fiber composite 
material is applied to the non-planar structure, the strain driven becomes non-directional (the induction of the vibration signal is also non-directional), and all the strain energy density becomes low. In order to overcome these shortcomings, in 1999, Ying Luo, who comes from Jiangsu University, proposed the concept of 1-1-type piezoelectric orthotropic sensor components with the use of piezoelectric orthotropic composite components of the sensor in the two-dimensional and three-dimensional stress field decomposition of the stress wave component. The results obtained by domestic and foreign counterparts were of widespread concern and praise [13-15]. In 2000, an orthotropic nature of the piezoelectric composite material was developed in the United States, at the Massachusetts Institute of Technology, at its smart materials and structural laboratory $[16,17]$. The research and application of $1-3$ piezoelectric composite have become the research of piezoelectric composite materials both at home and abroad [18-20]. And they rarely study the research and application of 1-1 type piezoelectric composite materials. In recent years, the author group who developed the 1-1-type orthotropic piezoelectric composite material (OPCM) sensor, which was first used for engineering structural damage identification active monitoring [21-23], had made an achievement. This group has developed an S-type piezoelectric micro-displacement amplifier actuator and micro-band orthogonal anisotropic - acoustic emission sensor [24], and upon verification, its drive performance was better than that of conventional PZT actuator.

Based on the force-electric coupling theory, an OPCM element with telescopic vibration is constructed. The influence of the characteristics of the phase material and the structure size on the performance of the OPFC drive element is analyzed by CONSOL finite element simulation platform, and its optimal parameters are obtained, which provide guidance for further research on the new ultrasonic phased array transducer.

\section{The principle of piezoelectric actuator}

The schematic of a 1-1-type piezoelectric composite is as shown in Figure 1. In piezoelectric composite materials using transverse piezoelectric effect, the direction of polarization of each piezoelectric plate is parallel to the direction of the applied electric field, and it is along the thickness direction of the piezoelectric composite. The coordinate axis $\mathrm{z}$ is consistent with the polarization direction of the piezoelectric phase, the $\mathrm{x}-\mathrm{y}$ plane is perpendicular to the polarization direction, the electrode surface of the composite material is the $x-y$ surface, and the PZT material is a transversely isotropic material.

When the piezoelectric element acts as an actuator, it works on the basis of the inverse piezoelectric effect and generates electric strain under the action of an external electric field. The piezoelectric effect is expressed by the inverse piezoelectric effect, and the applied electric field is in three directions. If the structure of the bonded piezoelectric element is in the $1-3$ plane $(x-z$ plane, consistent with the 1-1-type piezoelectric composite material), as shown in Figure 2, the single-sided adhesive form is adopted. In the figure, $t_{\mathrm{b}}$ is the thickness of the elastic

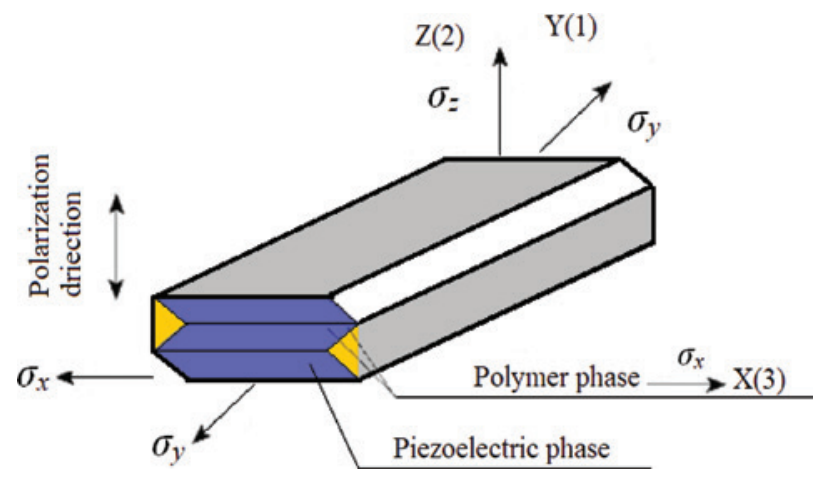

Figure 1: 1-1 OPCM longitudinal actuator element.
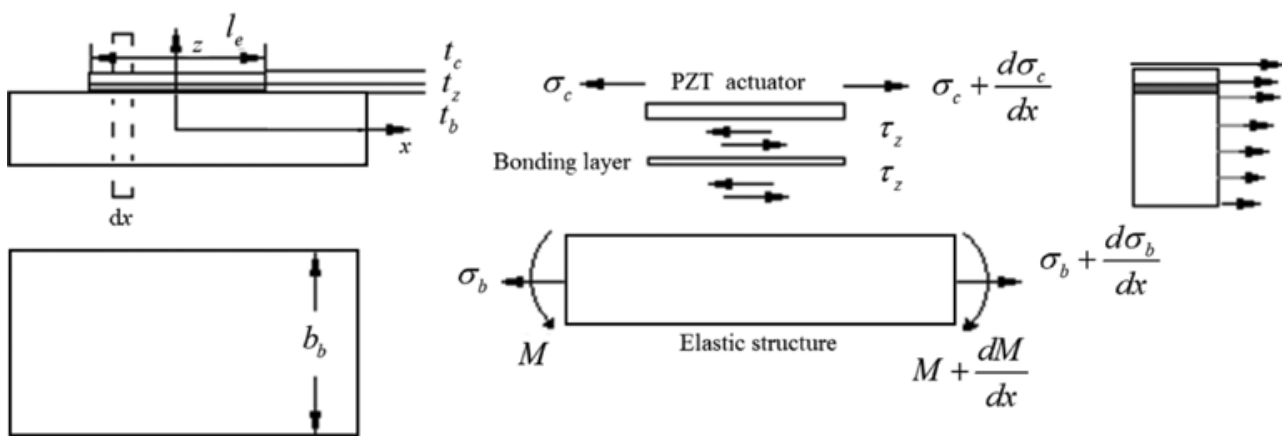

Figure 2: The actuating model of piezoelectric material. 
structure, $t_{\mathrm{z}}$ is the thickness of the adhesive layer, $t_{\mathrm{c}}$ is the thickness of the piezoelectric actuator, $l_{\mathrm{e}}$ is the length of the piezoelectric actuator, and $b_{\mathrm{b}}$ is the width of the elastic structure. When the OPCM element is used as an actuator, the static equilibrium equation of the actuator element can be expressed as follows:

$$
\frac{\partial \sigma_{o}}{\partial x}-\frac{\tau_{p}}{t_{c}}=0
$$

In the formula, $\sigma_{0}$ and $\tau_{\mathrm{p}}$ are the normal stress and shear stress of the actuator element, respectively.

The static balance equation of the elastic host component can be expressed as follows:

$$
\frac{\partial \sigma_{b}^{a}}{\partial x}-\frac{b_{c}}{b_{b} t_{b}} \tau_{p}=0
$$

In the formula, $b_{c}$ is the drive element width.

The moment balance equation of a piezoelectric composite drive element can be expressed as follows:

$$
\tau_{p} d x b_{o} \times \frac{1}{2} t_{b}+d M=0
$$

In the formula, $b_{0}$ is the thickness of the adhesive layer.

The torque at point A can be expressed as follows:

$$
\begin{gathered}
M=\int_{-\frac{1}{2} t_{b}}^{\frac{1}{2} t_{b}} \sigma_{y} b_{b} d y \\
M=\frac{1}{6} b_{b} t_{b}^{2}\left(\sigma_{b}^{p}-\sigma_{b}^{o}\right)
\end{gathered}
$$

In the formula, $\sigma_{b}^{p}$ and $\sigma_{b}^{0}$ are the normal stresses of the coupling layer and the host component, respectively.

Substituting formula (5) with formula (3), the moment balance equation of the host component can be obtained:

$$
\frac{3 b_{c}}{b_{b} t_{b}} \tau_{p}+\frac{\partial \sigma_{b}^{p}}{\partial x}-\frac{\partial \sigma_{b}^{o}}{\partial x}=0
$$

In this mechanical model, the stress-strain relationship in the piezoelectric composite actuator, the coupling layer, and the host structure can be expressed as follows:

$$
\varepsilon_{o}=\frac{\partial u_{o}}{\partial x} \quad \varepsilon_{b}^{p}=\frac{\partial u_{b}^{p}}{\partial x} \quad \varepsilon_{b}^{o}=\frac{\partial u_{b}^{o}}{\partial x} \quad r_{p}=\frac{u_{o}-u_{b}^{p}}{t_{p}}
$$

In the formula, $\varepsilon_{0}$ and $u_{0}$ are, respectively, the linear strain and displacement components of the actuator element; $\varepsilon_{b}^{p}$ and $u_{b}^{p}$ are the linear strain and displacement components of the coupling layer; $\varepsilon_{b}^{0}$ and $u_{b}^{0}$ are, respectively, the linear strain and displacement components of the host component, and $r_{p}$ is the shear strain.

The stress-strain relationship of piezoelectric composites can be obtained by the inverse piezoelectric equations:

$$
\sigma_{o}=E_{o}\left(\varepsilon_{p}-\frac{e_{31}}{E_{c}} E_{3}\right)
$$

The stress-strain relationship in the host component elastic material can be expressed as follows:

$$
\sigma_{b}^{p}=E_{b} \varepsilon_{b}^{p} \quad \sigma_{b}^{o}=E_{b} \varepsilon_{b}^{o} \quad \tau_{p}=G r_{p}
$$

In the formula, $e_{31}$ is the piezoelectric strain constant in one direction; $E_{0}, E_{c}$, and $E_{b}$ are the elastic moduli of the host component, the piezoelectric material, and the coupling layer, respectively; $E_{3}$ is the piezoelectric phase elastic modulus; and $G_{p}$ is the shear modulus of the piezoelectric material.

Substituting formula (9) with formula (6), the following equations can be obtained:

$$
\begin{gathered}
\frac{\partial^{2} \varepsilon_{o}}{\partial x^{2}}-\frac{G_{p}}{E_{o} t_{o} t_{p}}\left(\varepsilon_{o}-\varepsilon_{b}^{p}\right)=0 \\
\frac{\partial^{2} \varepsilon_{b}^{p}}{\partial x^{2}}-\frac{\partial^{2} \varepsilon_{b}^{o}}{\partial x^{2}}+\frac{3 b_{o} G_{p}}{E_{b} t_{b} b_{b} t_{p}}\left(\varepsilon_{o}-\varepsilon_{b}^{p}\right)=0 \\
\frac{\partial^{2} \varepsilon_{b}^{p}}{\partial x^{2}}+\frac{b_{o} G_{p}}{E_{b} t_{b} b_{b} t_{p}}\left(\varepsilon_{o}-\varepsilon_{b}^{p}\right)=0
\end{gathered}
$$

It can be substituted by using the infinitude no dimension method:

$$
\begin{gathered}
\Gamma^{2}=\frac{\psi_{p}}{T_{p}^{2}}\left(\frac{\psi_{b}+4}{\psi_{p}}\right) \psi_{b}=\frac{E_{b} t_{b} b_{b}}{E_{o} t_{o} b_{o}} \quad \psi_{p}=\frac{G_{p} t_{p} b_{p}}{E_{o} t_{o} b_{o}} \\
T_{p}=\frac{t_{p}}{t_{o} / 2} \bar{x}=\frac{x}{l_{p} / 2}
\end{gathered}
$$

The boundary conditions of differential equation (11) can be expressed as follows:

$$
\varepsilon( \pm 1)=\Lambda=\frac{e_{31}}{E_{p}} E_{3} \quad \varepsilon_{b}^{p}( \pm 1)=0 \quad \varepsilon_{b}^{o}( \pm 1)=0
$$

In the formula, $\Lambda$ is the induced strain of the composite.

To solve the differential equations (15), 


$$
\begin{gathered}
\frac{\varepsilon_{c}(\bar{x})}{\wedge}=\frac{4}{\psi_{b}+4}+\frac{\psi_{b}}{\left(\psi_{b}+4\right) \cosh (\Gamma)} \cosh (\Gamma \bar{x}) \\
\frac{\varepsilon_{c}^{s}(\bar{x})}{\wedge}=\frac{4}{\psi_{b}+4}+\frac{4}{\left(\psi_{b}+4\right) \cosh (\Gamma)} \cosh (\Gamma \bar{x}) \\
\frac{\varepsilon_{c}^{o}(\bar{x})}{\wedge}=\frac{4}{\psi_{b}+4}+\frac{1}{\left(\psi_{b}+4\right) \cosh (\Gamma)} \cosh (\Gamma \bar{x}) \\
\frac{\tau_{s}}{\wedge}(\bar{x})=\frac{b_{b} t_{b} l_{c} E_{b} \Gamma}{2 b_{c}} \frac{\sinh (\Gamma \bar{x})}{\left(\psi_{b}+4\right) \cosh (\Gamma)}
\end{gathered}
$$

Formulas (16) and (17) are the strain distribution of the piezoelectric composite actuator and the elastic structure and the distribution of the shear stress of coupling layer, respectively.

\section{Structural parameters optimization of OPCM element}

\subsection{Effect of piezoelectric phase material properties on the component performance}

In the longitudinal wave actuator/sensor element structure of 1-1-type OPCM, the piezoelectric phase material played a leading role for the OPCM. Taking into account that the OPCM components require the best driving and sensing performance, piezoelectric phase materials and polymer phase materials were selected, which are the PZT-51 piezoelectric ceramic materials and DAD-40 epoxy resin conductive adhesive. The main parameters of the piezoelectric phase material include elastic modulus, Poisson's ratio, dielectric constant, etc., and the material parameters are as shown in Table 1. The total thickness of the OPCM element is $3.1 \mathrm{~mm}$, the length is $14 \mathrm{~mm}$, the width is $1.5 \mathrm{~mm}$, the thickness of the conductive adhesive is $0.1 \mathrm{~mm}$; the DAD-40 type epoxy conductive adhesive (room-temperature shear strength is $14.7 \mathrm{Mpa}$, the volume resistivity is less than $1.0 \times 10^{-3} \Omega \mathrm{cm}$ ) and these materials were configured as a strip-shaped cross-over electrode. When the OPCM element is driven, the voltage applied in the $\mathrm{z}$-direction is $10 \mathrm{~V}$.

The section size of the piezoelectric phase material is $1.5 \mathrm{~mm} \times 0.7 \mathrm{~mm}$, and the section size of the polymer phase material is $1.5 \mathrm{~mm} \times 0.1 \mathrm{~mm}$. The model of OPCM longitudinal wave actuator element that was established after inputting the corresponding material parameters is as shown in Figure 3.
Figure 4 shows the stress distribution of the 1-1 longitudinal wave OPCM element under the action of electric displacement $D=140 \mathrm{C} / \mathrm{m}^{2}$. In the OPCM piezoelectric composites, the elastic modulus of the polymer phase material is much smaller than that of the piezoelectric phase material. When the piezoelectric composite is subjected to external stress, the stress acting on the polymer phase material will be transmitted to the piezoelectric phase material, resulting in a stress amplification effect on the piezoelectric phase material, that is, the stress-focus phenomenon. At the same time, the dielectric constant of the polymer phase is extremely low, which makes the overall dielectric constant of the piezoelectric composite decrease greatly. For these two reasons, the piezoelectric composition $\mathrm{g}$ of the piezoelectric composite is greatly improved. The addition of the polymer also improves the flexibility of the piezoelectric composite, resulting in a significant improvement in its comprehensive properties.

\subsection{Effect of elastic modulus of piezoelectric phase on the performance of element}

According to the constitutive relation of the superimposed piezoelectric composite, the piezoelectric elastic

Table 1: The basic material parameter of PZT-51.

\begin{tabular}{llllllllllll}
\hline $\begin{array}{l}\text { Density } \\
\left(\mathbf{k g} / \mathbf{m}^{3}\right)\end{array}$ & $\begin{array}{l}\text { Piezoelectric } \\
\text { constant } 10^{-12} \\
\mathbf{C} / \mathbf{N}\end{array}$ & $\begin{array}{l}\text { Compliance } \\
\text { constant } \\
\mathbf{m}^{2} / \mathbf{N}\end{array}$ & $\begin{array}{l}\text { Poisson } \\
\text { ratio }\end{array}$ & $\begin{array}{l}\text { Dielectric } \\
\text { constant }\end{array}$ \\
\hline$\rho$ & $d_{31}$ & $d_{33}$ & $d_{15}$ & $S_{11}^{E}$ & $S_{33}^{E}$ & $S_{55}^{E}$ & $\mu_{12}$ & $\mu_{13}$ & $\varepsilon_{33}^{T}$ & $\varepsilon_{11}^{T}$ \\
450 & -185 & 400 & 650 & 15 & 19 & 22 & 0.32 & 0.6 & 2100 & 2400 \\
\hline
\end{tabular}

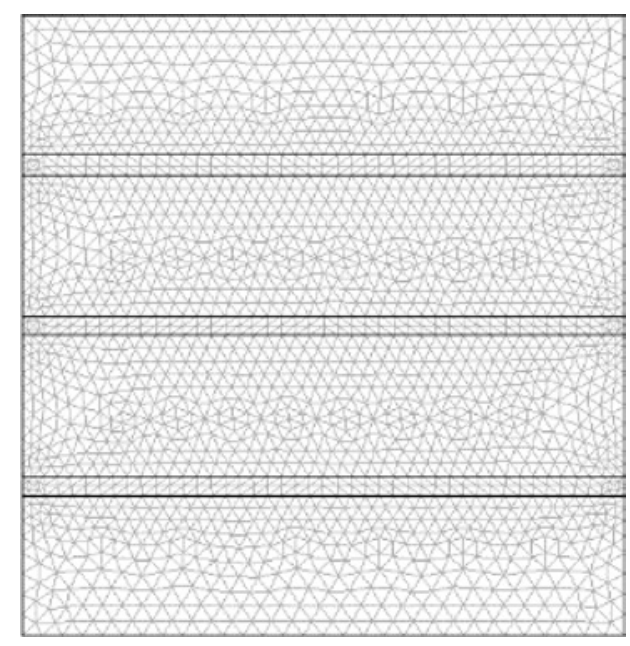

Figure 3: Finite model of OPCM longitude actuator element. 


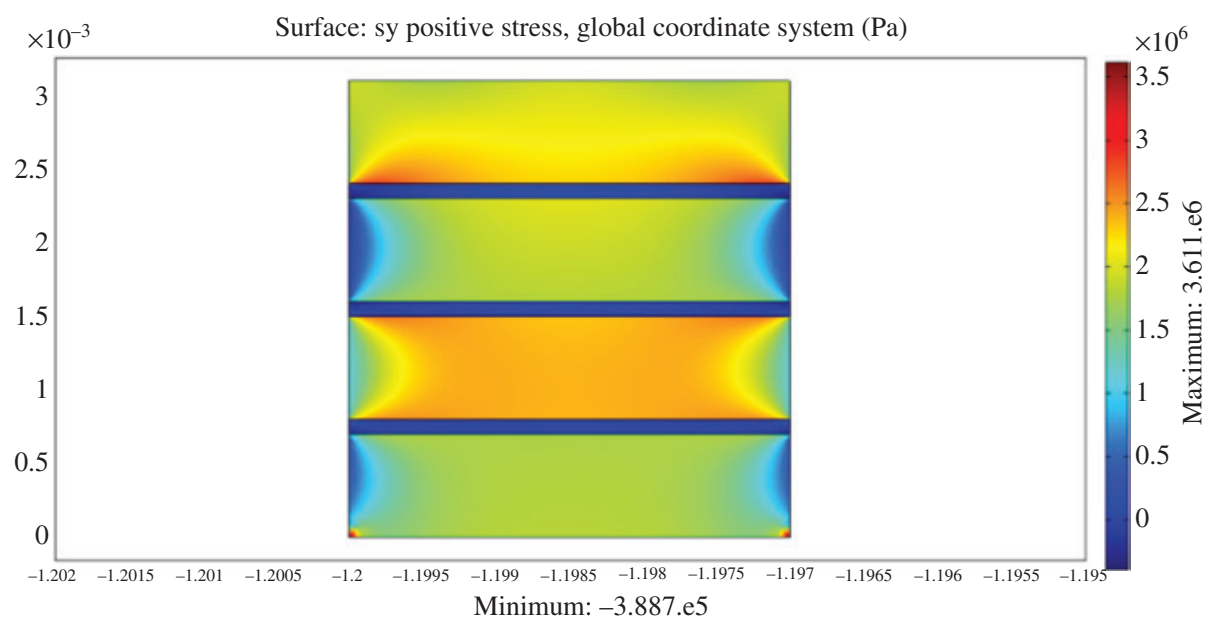

Figure 4: Charging initial electric displacement $\left(D=140 \mathrm{C} / \mathrm{m}^{2}\right)$.

modulus $E_{3}$ has a certain influence on the piezoelectric properties and mechanical properties of the whole piezoelectric composite. It is easy to find the effect law of $E_{3}$ range from $6.8 \times 10^{9}$ to $8.2 \times 10^{9}$ on the driving performance of the OPCM element from Figure 5. With the increase of the elastic modulus, the strain of the OPCM element in the $\mathrm{z}$-direction decreases, but the strain in the $\mathrm{x}$-direction does not change much. For the same elastic modulus, when the applied voltage is $10 \mathrm{~V}$, the strain in the $\mathrm{z}$ direction is significantly larger than that in the $\mathrm{x}$ direction, which also shows that the piezoelectric composite has obvious orthotropic component, and as the piezoelectric modulus increases, the strain tends to decrease for the same voltage applied.

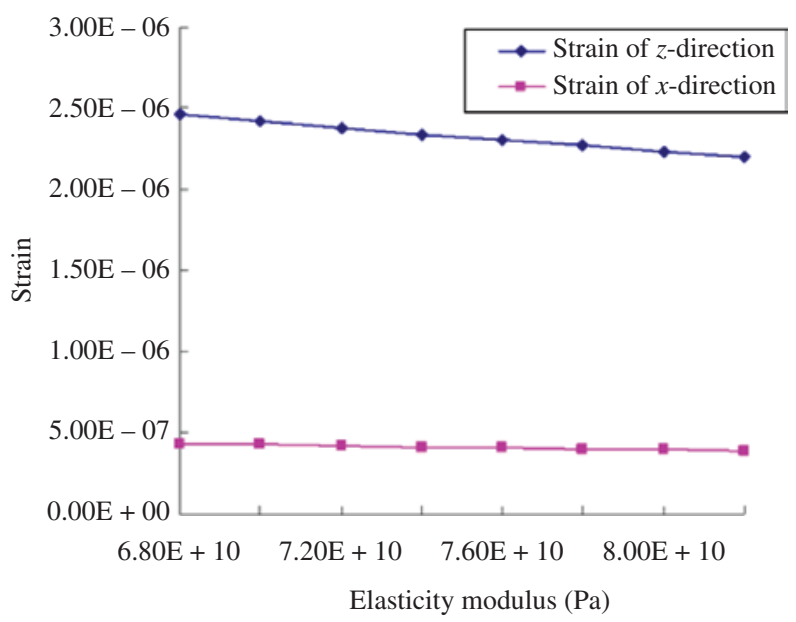

Figure 5: Relationship between piezoelectric elastic modulus and strain of composite material $(U=10 \mathrm{~V})$.

\subsection{Effect of dielectric constant of piezoelectric phase on component performance}

As the dielectric constant of the polymer phase in the piezoelectric composite material is very low, the dielectric constant of the entire OPCM element is mainly determined in the piezoelectric phase. Figure 6 shows the effect of the dielectric constant of the piezoelectric phase on the driving performance of the composite. It can be seen that with the increase of the dielectric constant, the strain gradually increases, and the variation in the $y$ direction is not significant. According to the electrostatic field formula and the orthogonal anisotropic, selecting the piezoelectric phase material with large dielectric

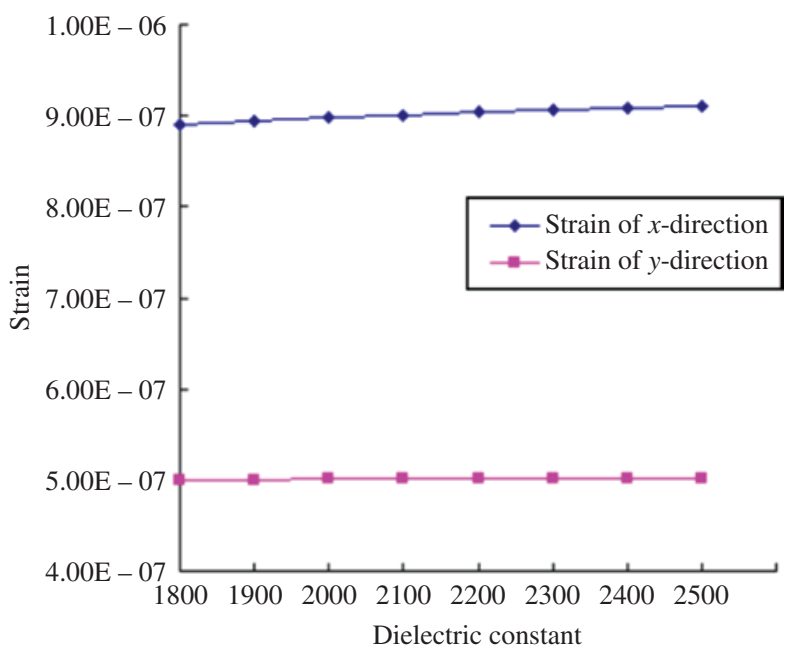

Figure 6: Relationship between piezoelectric dielectric coefficient and strain of composite material. 
constant can improve the actuating performance of the OPCM driving element.

\subsection{Effect of Poisson's ratio of piezo- electric phase on the performance of components}

From the constitutive relation of 1-1-type piezoelectric composites, it can be seen that the Poisson's ratio at the piezoelectric phase has certain influence on the piezoelectric properties and mechanical properties of OPCM, as shown in Figure 7. With the increase of the Poisson's ratio of the piezoelectric phase material, the induction strain of the OPCM driving element is increasing, but the variation is not significant, which indicates that the piezoelectric material with high Poisson's ratio is helpful to improve the deformation performance of the OPCM longitudinal wave drive element. For the same Poisson's ratio, the strain of the OPCM element in $\mathrm{z}$ direction is 5.6 times the strain in the $\mathrm{x}$ direction, which also indicates that the piezoelectric composite structure has orthotropic properties.

\subsection{Effect of elastic modulus of polymer phase on component performance}

When the OPCM element is used as a longitudinal wave actuator, the piezoelectric phase material generates an induced strain under the action of an electric field, which is then transferred to the host component through the polymer phase material, so selecting the appropriate polymer phase material contributes to ensuring and improving the performance of the piezoelectric composites.

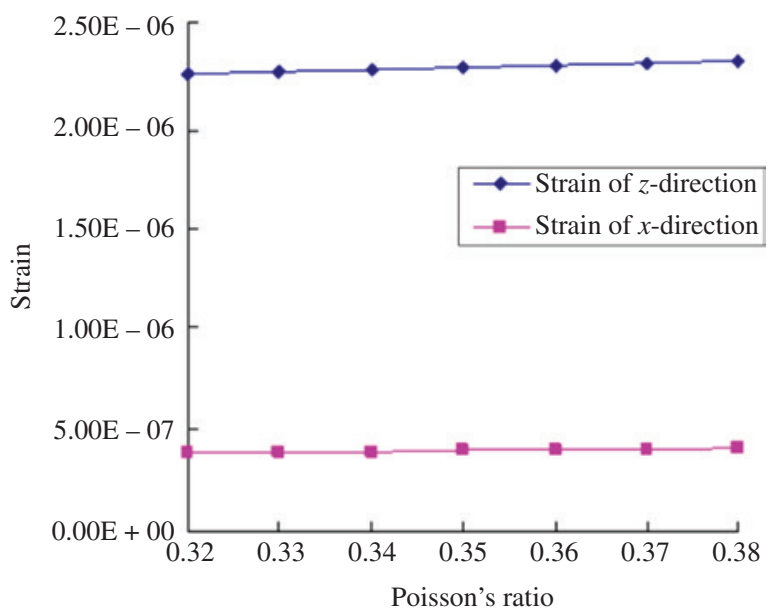

Figure 7: Relationship between piezoelectric Poisson's ratio and strain of composite material $(U=10 \mathrm{~V})$
The influence of the elastic modulus of the polymer phase on the properties of the composite is as shown in Figure 8. The strain does not change much as the elastic modulus of the polymer increases. It is easy to enhance the stress-focus phenomenon of piezoelectric phase by choosing a low elastic modulus polymer phase material, thereby enhancing the overall performance of OPCM longitudinal wave drive components. Therefore, selecting the low elastic modulus of the polymer can improve the composite performance when designing and manufacturing.

\subsection{Effect of Poisson's ratio of polymer phase on component performance}

The effect of Poisson's ratio on the properties of piezoelectric composites is as shown in Figure 9. The Poisson's ratio of polymer phase has little effect on the properties of the composites, and the tendency of strain to decrease is small. The strain in the $\mathrm{x}$ direction is 5.6 times the strain in the y direction, indicating that the OPCM element has obvious orthotropic properties. It also shows that, as the Poisson's ratio increases, the polymer increases the stress or strain amplification of the piezoelectric composite.

\section{Influence of geometric parameters on the performance of OPCM longitudinal wave actuating/ sensing element}

In addition to the material parameters on the performance of OPCM components, there are geometric structure

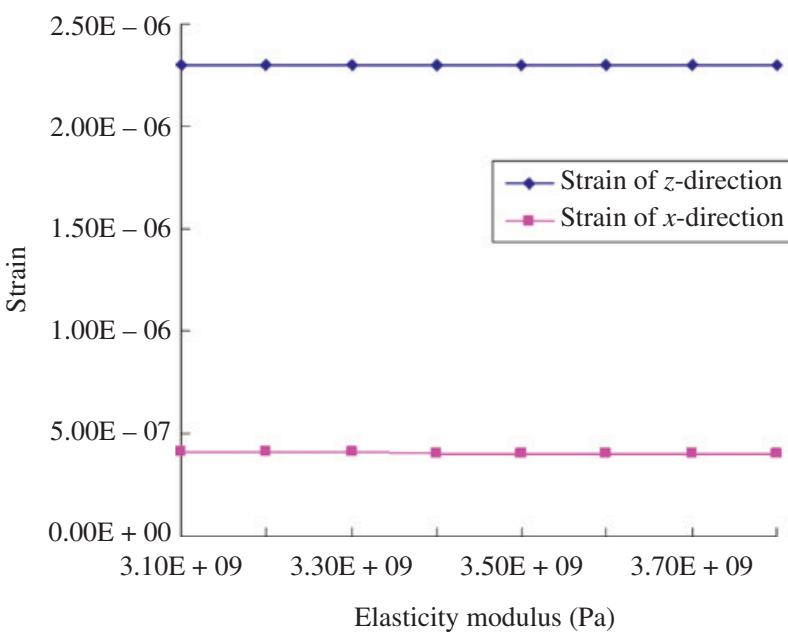

Figure 8: Relationship between polymer phase elastic modulus and strain of composite material $(U=10 \mathrm{~V})$. 


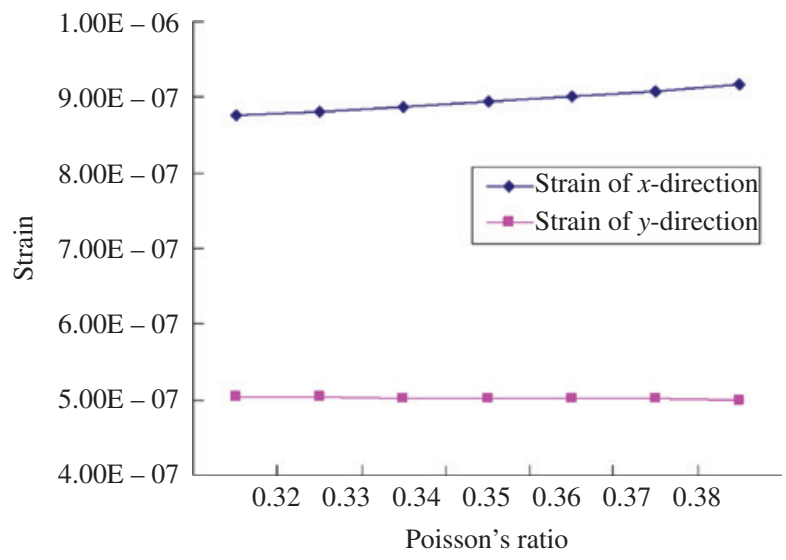

Figure 9: Relationship between polymer phase Poisson's ratio and strain of composite material $(U=10 \mathrm{~V})$.

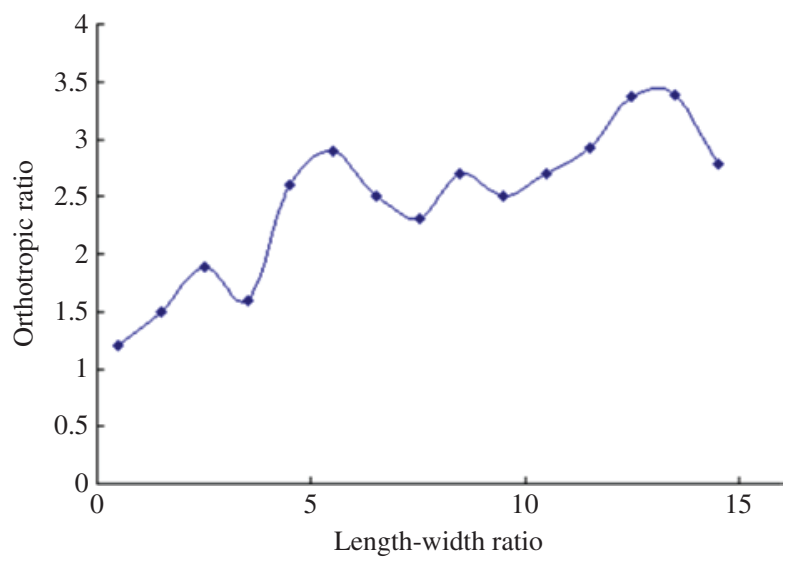

Figure 10: The relationship between the aspect ratio and the orthotropic ratio.

parameters that also affect the performance of OPCM components by analyzing the different OPCM drive/sensor components aspect ratio and thickness and its impact on the performance.

\subsection{Influence of piezoelectric phase to long ratio on orthotropic performance}

When the OPCM element is driven, an electric field voltage of $10 \mathrm{~V}$ is applied in the direction of polarization of the piezoelectric phase material to extract the strain values in the $\mathrm{x}$ direction and the $\mathrm{z}$ direction, respectively. It can be seen from Figure 10 that increasing the aspect ratio of the piezoelectric phase material can increase the orthogonality ratio of the OPCM element. The polarization direction is in the $\mathrm{z}$ direction; the performance in the polarization direction and the performance in the other two directions are on the contrary, and just to meet the Poisson's effect of the material, when the aspect ratio is 14 , which is the orthotropic ratio to reach the maximum combined with the processing technology, we used the aspect ratio as the best study size.

\subsection{Effect of thickness on orthotropic properties of composites}

As shown in Figure 11, the thickness of the OPCM element changes its performance. When the thickness of the OPCM element with a length of $14 \times 1 \mathrm{~mm}$ changes from $0.7 \mathrm{~mm}$ to $3.1 \mathrm{~mm}$, the OPCM longitudinal wave drive element, the resonant frequency decreases rapidly. After $100 \mathrm{kHz}$, it tends to be gentle, and when combined with the test material in this experiment, it requires frequency analysis to select the four-layer piezoelectric film structure, which is more appropriate. Figure 12 shows the effect of the aspect ratio on the resonant frequency. It can be seen that when the aspect ratio is 2.1, the frequency is the largest, so the OPCM element structure of the frequency segment can be selected as an actuator.

The influence of the physical parameters and geometrical parameters on the driving performance of the OPCM element is analyzed. The optimal selection of PZT piezoelectric phase and polymer phase material and the OPCM element with the best thickness and aspect ratio for the experimental scheme are obtained. The OPCM components can produce directional stress wave signal and are conducive to the concentration of directional energy, reducing the side of the signal generated in the interference. The OPCM for the waveguide drive components, due to the existence of orthotropic properties in the $\mathrm{x}$ direction and y direction of the excitation voltage, is not consistent; if used for the development of ultrasonic phased

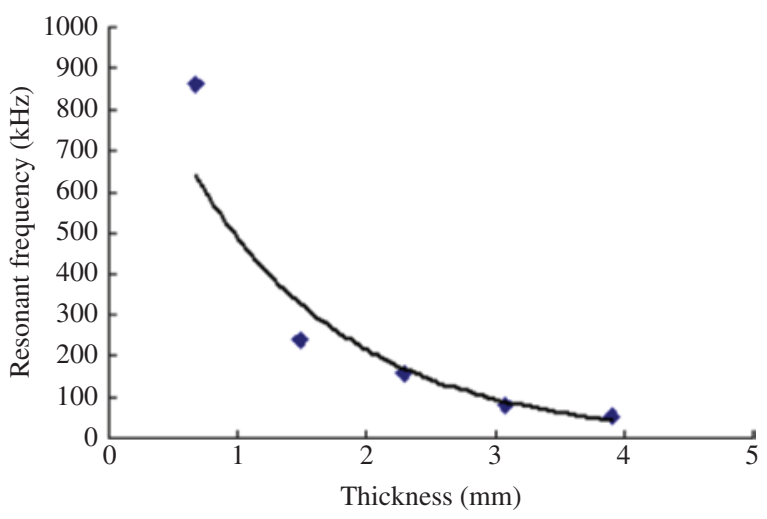

Figure 11: Relationship between thickness and resonant frequency. 


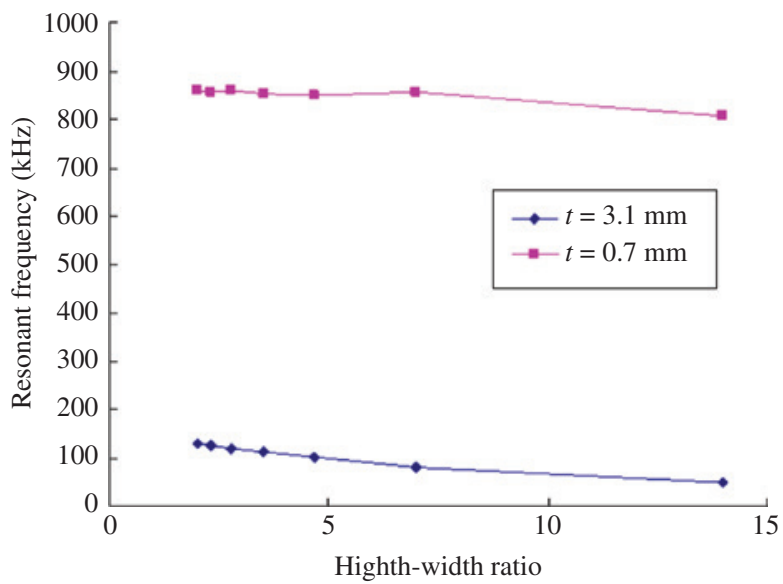

Figure 12: Relationship between aspect ratio and resonant.

array actuator, the delay will occur with the wavefront shape change, because the formation of the wave front is calculated by the arc, and the OPCM waveguide excitation signal formed by the elliptical line is not conducive to the calculation of different material delay law. So for OPCM guided wave drive components for a single actuator within the ultrasonic guided wave detection, only the OPCM P-wave drive/sensing element is used in the ultrasonic phased array detection technique of the engineering structure, which enhances the effective signal of the sensitivity direction emission and reduces the direction of the sensitive direction (lateral) of the signal to stimulate, so as to improve the signal-to-noise ratio for the engineering structure of ultrasonic phased array technology and to solve the problem of lateral reflection of the interference.

\section{Summary}

The force-electric coupling relationship and mechanical and electrical properties of 1-1-type piezoelectric composites have been the main factors to study and develop the piezoelectric composite materials in practice. The finite element method is used to analyze the piezoelectric phase, the polymer phase, and the geometric parameters. The influence of OPCM component driving and sensing performance and the best materials and geometric parameters are obtained. Among the various factors that influence OPCM, the piezoelectric properties of piezoelectric composites are mainly affected by the piezoelectric phase and are also affected by the polymer phase to a certain extent, and its mechanical properties are greatly influenced by the polymer phase.

Acknowledgments: The author(s) disclosed receipt of the following financial support for the research, authorship, and/or publication of this article: This work was financially supported by the National Natural Science Foundation of China (11872191 and 11520101001), The Key R\&D Plan of Jiangsu Province (BE2015138) and The Key R\&D Plan of Zhenjiang city (GY2015054) and Jiangsu University Foundation (14JDG022).

\section{References}

[1] Komura I, Hirasawa T, Nagai S, Takabayashi J, Naruse K. Nucl. Eng. Des. 2001, 206, 351-362.

[2] Chen CF, Han XL, Liu J, Luo Y. Nanosci. Nanotech. Lett. 2012, 4, 95-99.

[3] Cross LE. Mater. Chem. Phys. 1996. 43, 108-115.

[4] Newnham R, Safari A, Sa GG, Giniewicz J. Ferroelectrics 1984, $60,15-21$.

[5] Chan HW, Unsworth J. IEEE T. Ultrason. Ferr. 1989, 36, 434-441.

[6] Safari AA, Janas V, Jadidian B, French JD, Weitz GE, Luke JE. Proceedings of SPIE. 1996, 2721, 240-250.

[7] Gachagan A, Hayward G, Wright WMD, Hutchins DA. IEEE, 1993, 2, 651-654.

[8] Boland CR, Thibodeau SN, Hamiton SR, Sidransky D, Eshleman JR. Cancer Res. 1998, 58, 5248.

[9] Bent AA, Hagood NW, Rodgers JP. J. Intel. Mat. Syst. Str. 1995, 6, 338-349.

[10] Bent AA, Hagood NW. J. Intel. Mat. Syst. Str. 1997, 8, 903-919.

[11] Rossetti GJ, Pizzochero A, Bent A. IEEE 2000, 2, 753-756.

[12] Tan P, Tong L. Compos. Sci. Technol. 2001, 61, 759-769.

[13] Luo Y, Tao BQ. Acta Mech. Solida Sin. 2000, 13, 337-345.

[14] Saber N, Araby S, Meng QS, Hsu HY, Yan C, Azari, S, Lee SH, XU Y, Ma J, Yu S. Nanotechnology 2017, 25, 045501.

[15] Yousif H, Valerie JP, Richard EC, Paul DW, Chuan L. IEEE T. Ultrason. Ferr. 2013, 60, 1935-1948.

[16] Benjeddou A. Comput. Struct. 2000, 76, 347-363.

[17] Pettermann HE, Suresh S. Int. J. Solids Struct. 2000, 37, 5447-5464.

[18] Hossack JA, Hayward G. IEEE T. Ultrason. Ferr. 1991, 38, 618-629.

[19] Lous GM, Cornejo IV, Mcnulty TF, Safari A, Danforth SC. J. Am. Ceram. Soc. 2000, 83, 124-128.

[20] Ray MC, Pradhan AK. Mech. Adv. Matl. Stru. 2007, 14, 245-261.

[21] Liu QH, Zhu BY, Luo Y. J. Jiangsu Univ. 2006, 27, 172-175.

[22] Della CN, Shu DW. Acta Mater. 2008, 56, 754-761.

[23] Rao CVM, Prasad G. Condens. Matter Phys. 2010, 13, 1-10.

[24] Wang ZP, Luo Y, Zhao GQ, Yuan FG. Int. J. Mech. Mater. Des. 2017, 13, 57-69. 